\title{
MEASUREMENT OF FLOW RESISTIVITY USING COMPUTATIONAL FLUID DYNAMICS
}

\author{
Aamir Khan, Dr. S. Jeyanthi \\ Department of Mechanical and Building Science, VIT University, Chennai, India. \\ Hemantika Aggarwal \\ Department of Electrical and Instrumentation, Thapar Institute of Engineering and \\ Technology, Patiala, India \\ Karanbir Singh, Robin Kumar, Shivam Bhatia \\ Department of Mechanical and Building Science, VIT University Chennai, India.
}

\begin{abstract}
Flow resistivity is one of the most important parameters used to describe the acoustic behavior of fibrous materials, as porosity is highly used in industrial context, this paper summarizes the simulation of finding the flow resistivity using computational fluid dynamics and minimizing the error between calculated and measured resistivity.

The estimation is made by taking Polyurethane Foam as sample fibrous material and varying porosity from 0.70 up to 0.98. The CFD tool analyzes and computes different pressure value respectively. Flow resistivity is further verified using experimental execution in which flow resistivity is measured from pressure measurement on a sample of porous material
\end{abstract}

Keywords: flow resistivity, fibrous material, CFD tool.

Cite this Article: Aamir Khan, Dr. S. Jeyanthi, Hemantika Aggarwal, Karanbir Singh, Robin Kumar and Shivam Bhatia, Measurement of Flow Resistivity using Computational Fluid Dynamics, International Journal of Mechanical Engineering and Technology 11(3), 2020, pp. 23-29.

http://iaeme.com/Home/issue/IJMET?Volume=11\&Issue=3

\section{INTRODUCTION}

The Air flow resistivity is a highly critical parameter used to design various machines such as heat exchanger, reactors, ion exchangers etc. Even sound propagation in porous materials depends upon flow resistivity. Hence, a widely used industrial parameter, there are various ways of determining flow resistivity.

Flow depends on numerous factors including direction of flow, permeability of the substance, cross sectional area along with many other parameters but primarily it depends on the structure of the material and the velocity of the fluid.

At lowlevels Reynolds number and the viscous forces are dominant so they can be used. 
In other cases Darcy's law can be successfully used [2].It is defined as the rate at which a fluid flow through a permeable medium, such as an aquifer, is directly proportional to the drop in elevation between two places in medium and inversely proportional to the distance between them.

$$
\mathrm{Q}=-\mathrm{kA}(\mathrm{Pb}-\mathrm{Pa}) / \mu \mathrm{L}
$$

Where, $\mathrm{Q}$ is the discharge $(\mathrm{m} 3 / \mathrm{s}), \mathrm{A}$ is the area of material (m3), Pbis the outlet pressure $(\mathrm{Pa}), \mathrm{Pa}$ is the inlet pressure $(\mathrm{Pa}), \mu$ is the dynamic viscosity of the fluid (kg.m-1 s-1), $\mathrm{L}$ is the length of pipe $(\mathrm{m}), k$ is the Darcian permeability $\left(\mathrm{m}^{2}\right)$.

Porous materials are highly complex and random therefore for estimating dynamics of resistivity CFD tool is used

Based on Navier-Stokes equation, it is a computational software used to visualize how gas and liquid flows along with its effect on passing object. Along with giving insights of the flow CFD can also be used to investigate phenomena at micro scale level in different porous substrate.

\section{EXPERIMENTAL SETUP}

The main objective of the experiment is to find the flow resistivity of material as well as pressure loss. For this the Experiment is conducted taking a PVC pipe of diameter of $70 \mathrm{~mm}$ and length $40 \mathrm{~cm}$. A porous material is placed in between the pipe of thickness $3 \mathrm{~cm}$. and two pressure gauges are attached at inlet and outlet.

As the velocity of air deflects while passing through porous material, therefore the intensity of flow changes pressure at inlet and outlet. Flow resistivity can be calculated as given below. As shown in table 1 , the value of flow resistivity changes with porosity.

Where,

$$
\sigma=(\Delta \mathrm{p}) / \mathrm{V}^{*} \mathrm{Dp}
$$

$\Delta \mathrm{p}=$ change in pressure (convert to Pa from Psi)

$\mathrm{V}=$ velocity

$\mathrm{Dp}=$ diameter of material

Table 1 Pressure and flow resistivity value

\begin{tabular}{|c|c|c|c|}
\hline Porosity & \multicolumn{2}{|c|}{ Pressure (Pascal) } & Flow resistivity (Ns/m4) \\
\hline 0.69 & 102406.6 & 101322.8 & 14416.3486 \\
\hline 0.72 & 102207.2 & 101324.2 & 17333.296 \\
\hline 0.75 & 102301.4 & 101315.4 & 19633.5414 \\
\hline 0.78 & 101918.9 & 101321.7 & 21506.4802 \\
\hline 0.81 & 101798.2 & 101325 & 22485.0766 \\
\hline 0.84 & 101846.5 & 101314.2 & 23351.2263 \\
\hline
\end{tabular}

\section{SIMULATIONS}

Poroacoustics domain in the Comsol software is used for quantified analysis of resistivity. A hollow cylinder with porous material at the center our model that is to be analyzed. Major inputs include air properties and flow rate. For defining the porous material properties, the zwikkerkosten model allows for porosity and hydraulic diameter of pores to construct and analyze the material without the need of calculating viscous resistance and inertial resistance as is the case in Ansys ICEM porous material steady flow analyses [3]. 
The air wave is input at $50 \mathrm{~Hz}$ frequency and with the calculated velocity. The input wave is allotted the pressure of $400 \mathrm{~Pa}$.

The main inputs of the software are porosity, thickness and flow rate whose values are given in table 2 .

Table 2 Porosity with constant thickness \& flow rate

\begin{tabular}{|c|c|c|}
\hline Porosity & Thickness & Flow rate \\
\hline 0.70 & $20 \mathrm{~mm}$ & 15 Liter $/ \mathrm{min}$ \\
\hline 0.73 & $20 \mathrm{~mm}$ & 15 Liter $/ \mathrm{min}$ \\
\hline 0.76 & $20 \mathrm{~mm}$ & 15 Liter $/ \mathrm{min}$ \\
\hline 0.79 & $20 \mathrm{~mm}$ & $15 \mathrm{Liter} / \mathrm{min}$ \\
\hline 0.81 & $20 \mathrm{~mm}$ & $15 \mathrm{Liter} / \mathrm{min}$ \\
\hline 0.84 & $20 \mathrm{~mm}$ & $15 \mathrm{Liter} / \mathrm{min}$ \\
\hline 0.87 & $20 \mathrm{~mm}$ & $15 \mathrm{Liter} / \mathrm{min}$ \\
\hline 0.9 & $20 \mathrm{~mm}$ & 15 Liter $/ \mathrm{min}$ \\
\hline 0.92 & $20 \mathrm{~mm}$ & 15 Liter $/ \mathrm{min}$ \\
\hline 0.94 & $20 \mathrm{~mm}$ & $15 \mathrm{Liter} / \mathrm{min}$ \\
\hline 0.96 & $20 \mathrm{~mm}$ & $15 \mathrm{Liter} / \mathrm{min}$ \\
\hline
\end{tabular}

The value of flow rate is kept constant i.e 15 litre/min and porosity are varied at the rate of 0.03 starting from 0.69 up to 0.96 .

Velocity is further calculated using flow rate and area as given by

Velocity $=$ Flow rate/ Area

Area $=3.14 * \mathrm{R} * \mathrm{R}$

Radius $=45 \mathrm{~mm}$

Velocity $=0.0318 \mathrm{~m} / \mathrm{s}$

Hydraulic diameter of pore is also taken into account starting from $.00049 \mathrm{~m}$ and varying it according to porosity.

The model used is zwikker- kosten, whose input details are explained in figure 1.

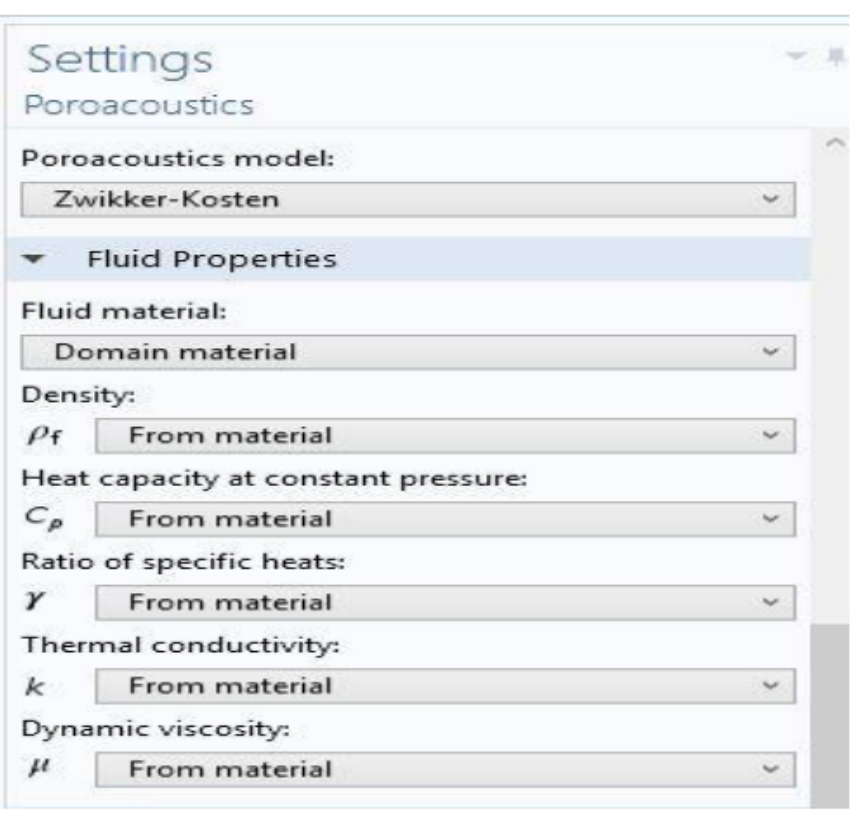

Figure 1 Snapshot of comsol software 


\section{RESULTS AND DISCUSSIONS}

Below figures gives the pressure value at inlet and outlet at different porosities. 12 simulations are prepared according to varying porosity with difference of 0.03 and for each value, pressure difference is obtained using CFD software, Resistivity is further calculated according to the given equation.

$$
\mathrm{R}=\mathrm{S} * \mathrm{P} / \mathrm{T} * \mathrm{U}
$$

Where,

$\mathrm{R}=$ Resistivity

$\mathrm{S}=$ Porosity

$\mathrm{P}=$ Pressure difference

$\mathrm{T}=$ Thickness

$\mathrm{U}=$ flow rate

The measured value and the experimental value of the resistivity has the error of only .001 . As porosity is directly proportional to the resistivity, therefore resistivity increases as porosity of the material increases

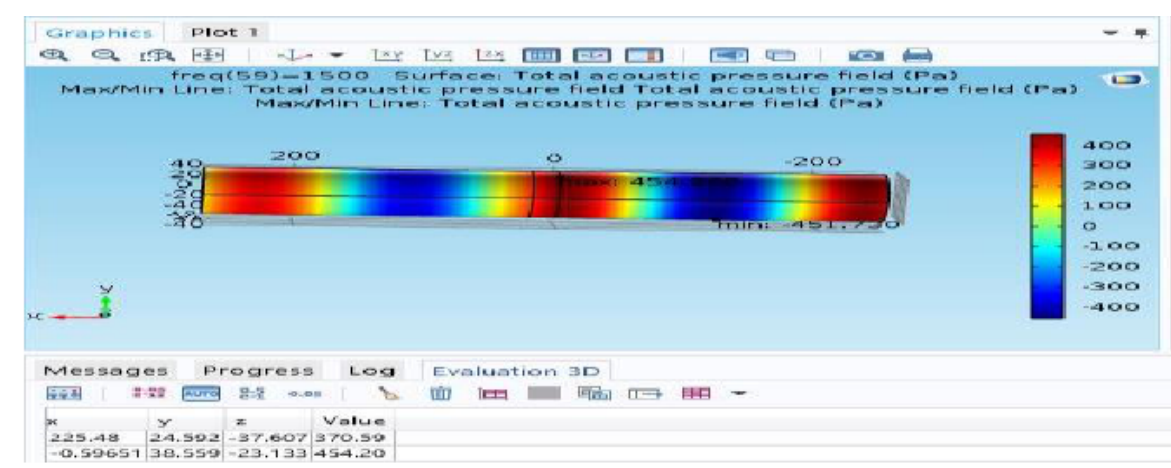

Figure 2 Pressure level at 0.70 porosity

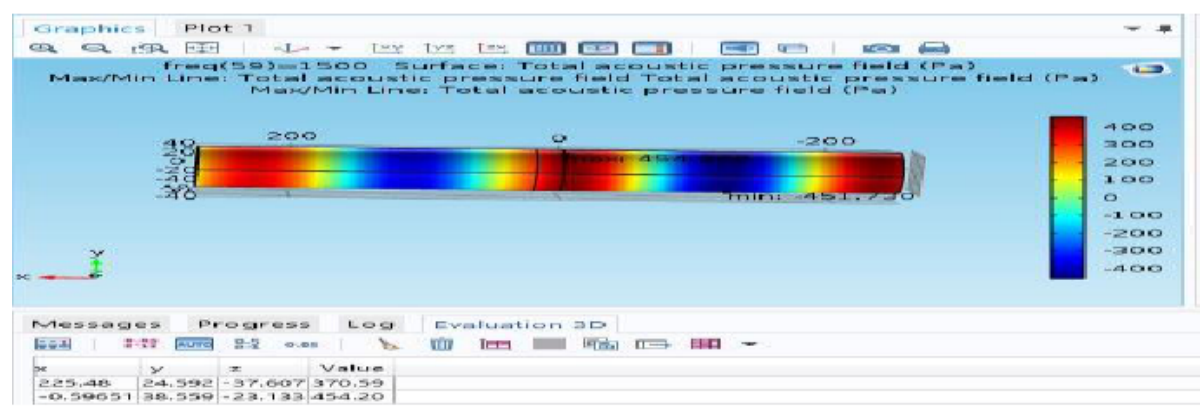

Figure 3 Pressure level at 0.73 porosity

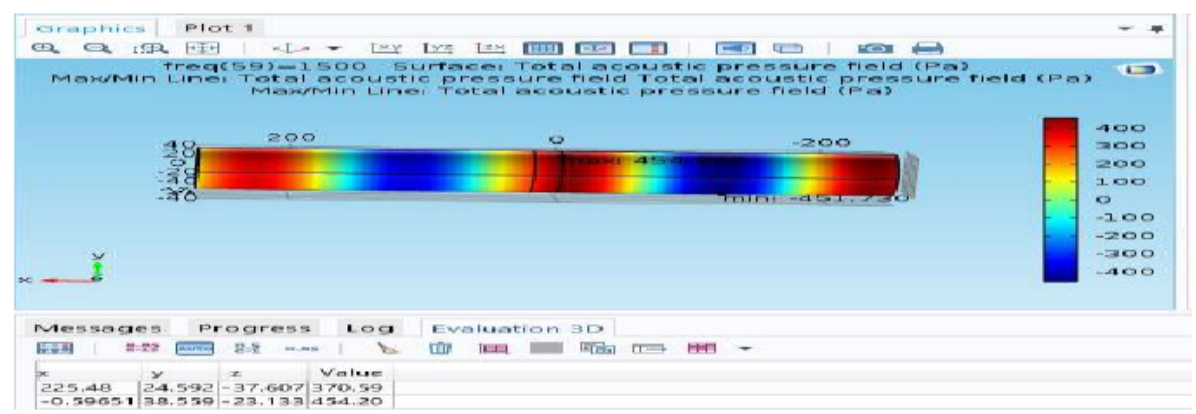

Figure 4 Pressure level at 0.76 porosity 


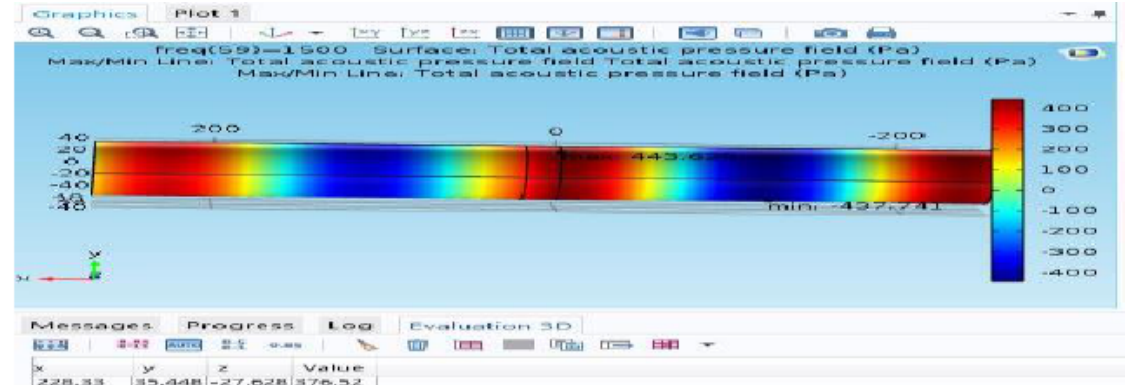

Figure 5 Pressure level at 0.79 porosity

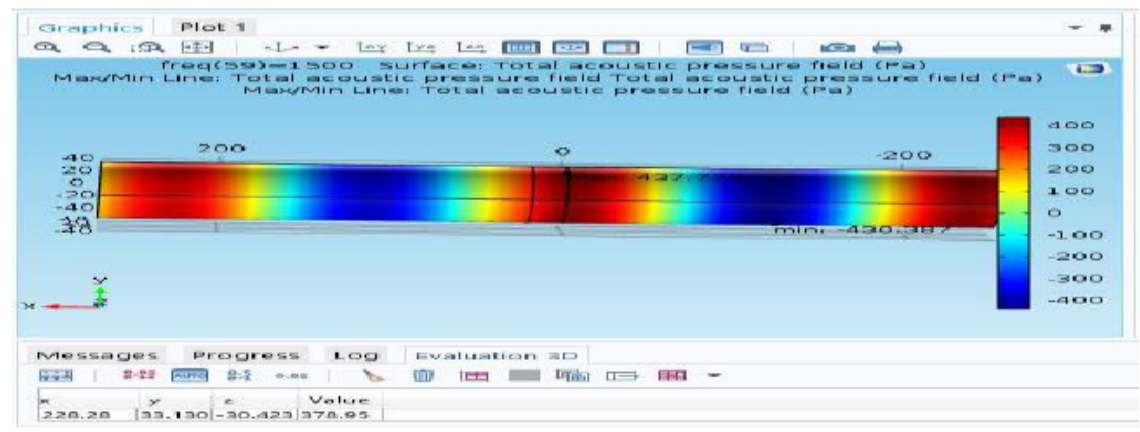

Figure 6 Pressure level at 0.81 porosity

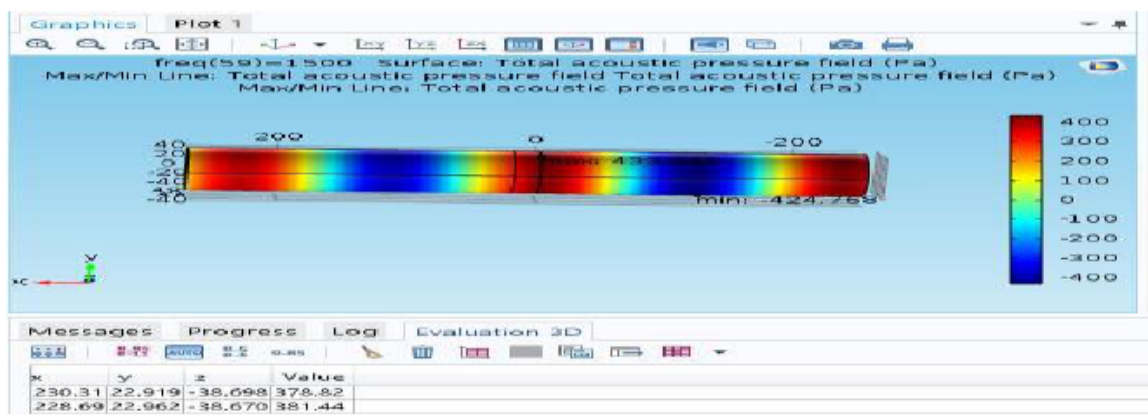

Figure 7 Pressure level at 0.84 porosity

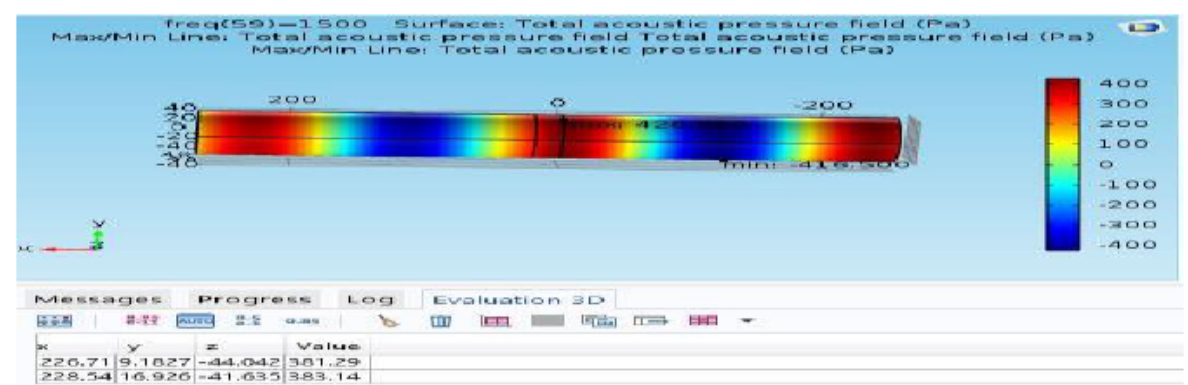

Figure 8 Pressure level at 0.87 porosity

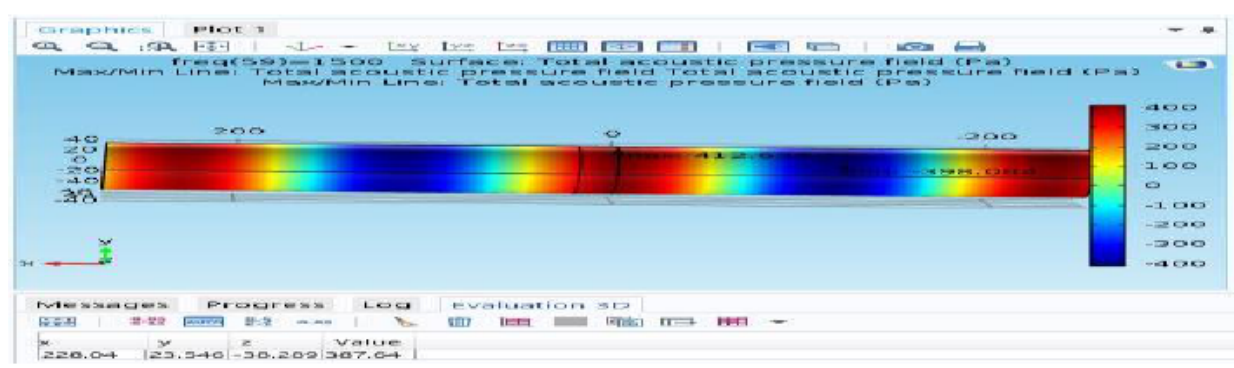

Figure 9 Pressure level at 0.90 porosity 
Aamir Khan, Dr. S. Jeyanthi, Hemantika Aggarwal, Karanbir Singh, Robin Kumar and Shivam Bhatia

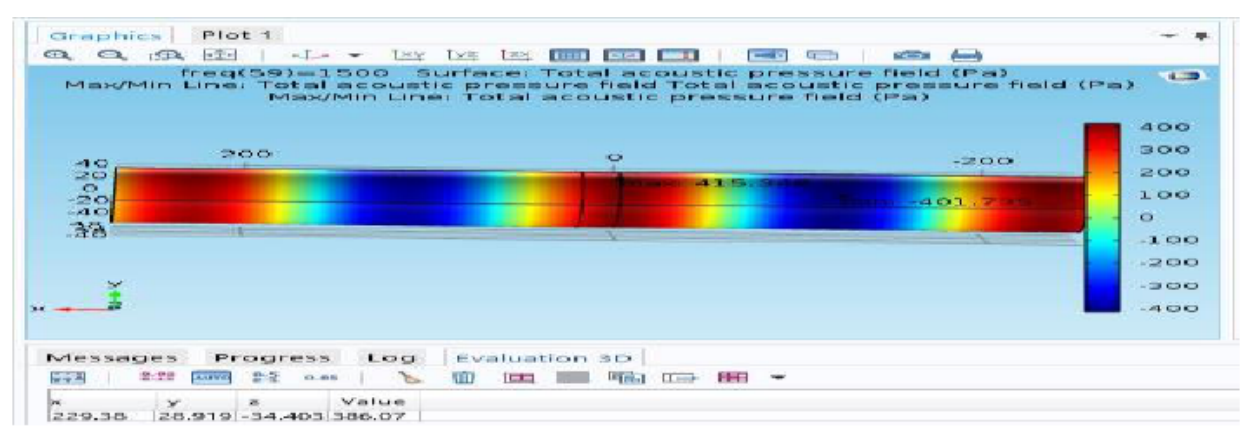

Figure 10 Pressure level at 0.92 porosity

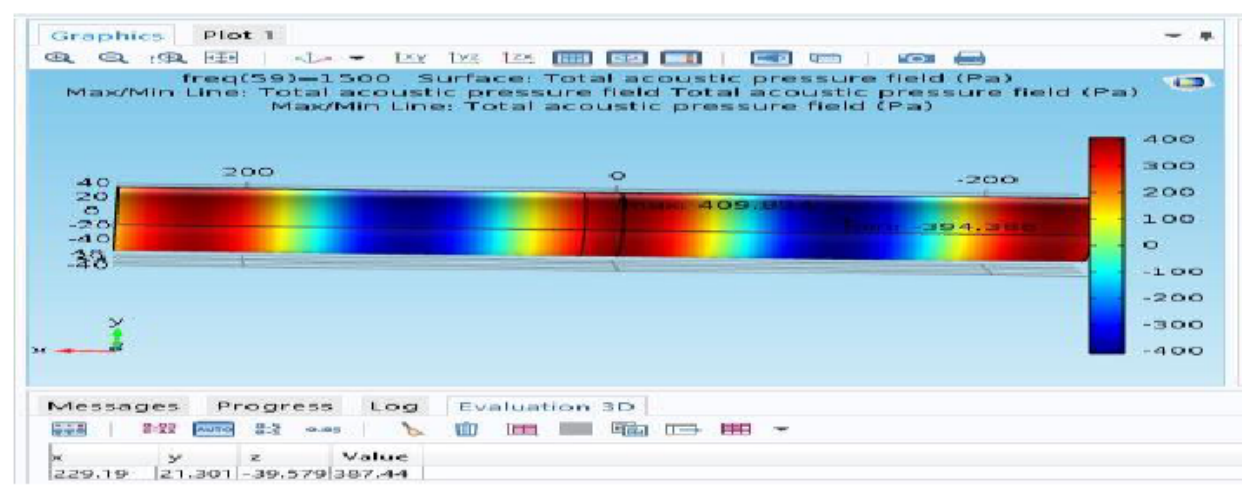

Figure 11 Pressure level at 0.94 porosity

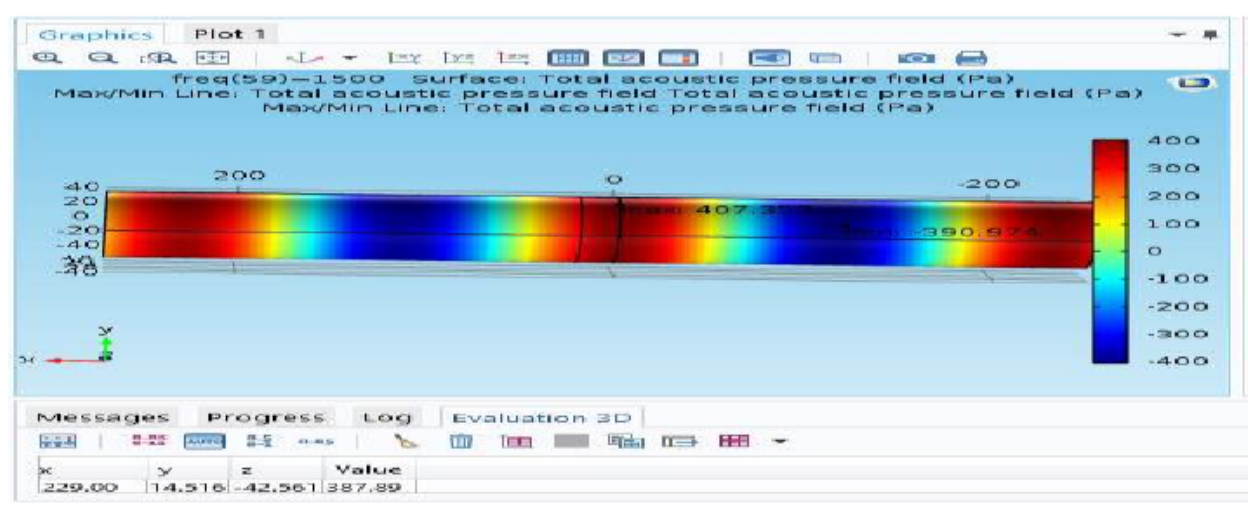

Figure 12 Pressure level at 0.96 porosity

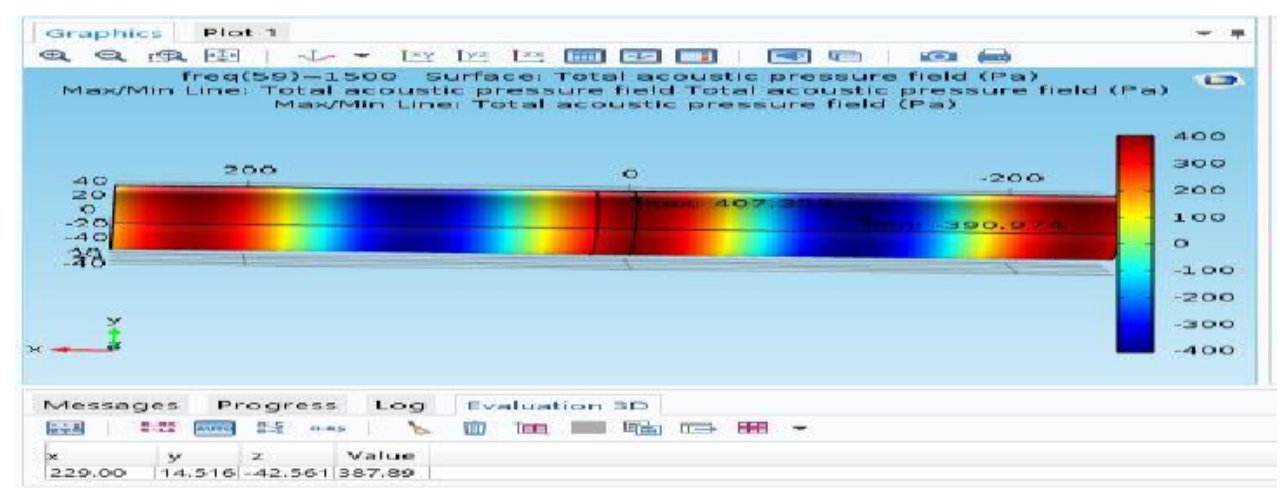

Figure 13 Pressure level at 0.96 porosity

Table 3 gives the value of pressure at inlet and outlet at each porosity level via computational fluid dynamics software and their respective resistivity can be easily calculated 
Table 3 Pressure and flow resistivity value

\begin{tabular}{|c|c|c|}
\hline Porosity & Pressure Difference & Resistivity \\
\hline 0.7 & $370-454$ & 131880 \\
\hline 0.73 & $373-450$ & 120890 \\
\hline 0.76 & $377-444$ & 105190 \\
\hline 0.79 & $379-438$ & 92630 \\
\hline 0.81 & $381-433$ & 81640 \\
\hline 0.84 & $382-428$ & 72220 \\
\hline 0.87 & $384-423$ & 61230 \\
\hline 0.9 & $385.5-418$ & 51025 \\
\hline 0.92 & $386-415$ & 45530 \\
\hline 0.94 & $387-412$ & 39250 \\
\hline 0.96 & $387.4-410$ & 35482 \\
\hline 0.98 & $388-407$ & 29830 \\
\hline
\end{tabular}

\section{CONCLUSION}

Flow resistivity have uncountable applications in engineering, hence purpose of this project was to characterize flow resistivity using CFD software and validate the results using experiments. The results are very accurate and precise to the experimental values with the error of only .001 Taking into account the anisotropic nature of material; there are small variations in the result which is also due to the deflection in thickness of porous material. The resistivity increases with increase in porosity as expected

\section{REFERENCES}

[1] H. G. Weller, G. Tabor, H. Jasak, C. Fureby, A Tensorial Approach to CFD using Object Orientated Techniques, Computers in Physics Vol. 12 (No. 6) (1998) 620

[2] Anderson J D 1995 Computational fluid dynamics. McGraw Hill, New York.

[3] G. Tabor, O. Yeo, P. Young, P. Laity, CFD Simulation of Flow through an Open Cell Foam, International Journal of Modern Physics Vol. 19 (2008) 703-715

[4] Amit Sharma., Mishra S.N.,Pradeep kumar and Bhanu Pratap Singh (2012) Development and thermal analysis of new class of storage elements International Journal of Engineering 